\title{
Highly Efficient Semiconductor-Based Metasurface for Photoelectrochemical Water Splitting: Broadband Light Perfect Absorption with Dimensions Smaller than the Diffusion Length
}

\author{
Amir Ghobadi ${ }^{1,2,3}$ (D) Turkan Gamze Ulusoy Ghobadi ${ }^{1,4,5} \cdot$ Ferdi Karadas $^{1,6} \cdot$ Ekmel Ozbay $^{1,2,3,7}$
}

Received: 12 September 2019 / Accepted: 4 December 2019 / Published online: 17 December 2019

(C) Springer Science+Business Media, LLC, part of Springer Nature 2019

\begin{abstract}
In this paper, we demonstrate a highly efficient light trapping design that is made of a metal-oxide-semiconductor-semiconductor (nanograting/nanopatch) $\left(\mathrm{MOSS}_{\mathrm{g} / \mathrm{p}}\right.$ ) four-layer design to absorb light in a broad wavelength regime in dimensions smaller than the hole diffusion length of the active layer. For this aim, we first adopt a modeling approach based on the transfer matrix method (TMM) to find out the absorption bandwidth (BW) limits of a simple hematite $\left(\alpha-\mathrm{Fe}_{2} \mathrm{O}_{3}\right)$-based metal-oxide-semiconductor (MOS) cavity design. Our modeling findings show that this design architecture can provide near-perfect absorption in shorter wavelengths. To extend the absorption toward longer wavelengths, a nanostructured semiconductor is placed on top of this MOS design. This nanostructure supports the Mie resonance and adds a new resonance in longer wavelengths without disrupting the lower wavelength absorption capability of MOS cavity. By this way, a polarization-insensitive absorption above 0.8 can be acquired up to $\lambda=565 \mathrm{~nm}$. Moreover, to have a better qualitative comparison, the water-splitting photocurrent of this design has been estimated. Our calculations show that a photocurrent as high as $10.6 \mathrm{~mA} \mathrm{~cm}^{-2}$ can be achieved with this design that is quite close to the theoretical limit of $12.5 \mathrm{~mA} \mathrm{~cm}{ }^{-2}$ for hematite-based water-splitting photoanode. This paper proposes a design approach in which the superposition of cavity modes and Mie resonances can lead to a broadband, polarization-insensitive, and omnidirectional near-perfect light absorption in dimensions smaller than the carrier's diffusion length. This can be considered as a winning strategy to design highly efficient and ultrathin optoelectronic designs in a variety of applications including photoelectrochemical water splitting and photovoltaics.
\end{abstract}

Keywords Metamaterials $\cdot$ Semiconductor metasurfaces $\cdot$ Perfect absorber $\cdot$ Plasmonics $\cdot$ Photochemistry $\cdot$ Light-driven water splitting

Amir Ghobadi

amir.ghobadi.ee@gmail.com

1 UNAM-National Nanotechnology Research Center, Bilkent University, 06800 Ankara, Turkey

2 NANOTAM - Nanotechnology Research Center, Bilkent University, 06800 Ankara, Turkey

3 Department of Electrical and Electronics Engineering, Bilkent University, 06800 Ankara, Turkey

4 Institute of Materials Science and Nanotechnology, Bilkent University, 06800 Ankara, Turkey

5 Department of Energy Engineering, Faculty of Engineering, Ankara University, 06830 Ankara, Turkey

6 Department of Chemistry, Bilkent University, 06800 Ankara, Turkey

7 Department of Physics, Bilkent University, 06800 Ankara, Turkey

\section{Introduction}

The concept of metamaterials and metasurfaces has emerged as a promising route to engineer the light-matter interaction in nanostructure geometries [1-7]. Light confinement in subwavelength geometries has turned into one of the most intensively explored areas in recent years. These devices are ideally designed to perfectly absorb and harvest light in a narrow or broad frequency range [8-23]. These perfect metamaterial absorbers have a wide range of applications including sensing [24-26], filtering [27, 28], coherent emission [29-32], photovoltaics and thermal photovoltaics [33-38], photodetection [39-41], solar vapor generation [42], and photochemistry [43-45]. Among these applications, photochemistry and photoelectrochemical water splitting has become a promising technology to supply the future clean energy demand, and it is thought to be the "holy grail" of energy conversion and storage revolution. For this reason, many different 
semiconductors and design configurations have proposed to achieve the required 10\% solar-to-hydrogen (STH) efficiency for commercialization of this technology in large-scale use [46-49]. The essential requirement for achieving this goal is to fully harvest the solar irradiation in a broad frequency range covering the visible (Vis) and near-infrared (NIR) regimes.

Among all different semiconductors, hematite $\left(\alpha-\mathrm{Fe}_{2} \mathrm{O}_{3}\right)$ with a narrow optical band gap $(1.9-2.1 \mathrm{eV})$ can reach to a maximum theoretical STH efficiency of $15 \%$ that corresponds to a photocurrent value of $12.5 \mathrm{~mA} \mathrm{~cm}^{-2}$. However, besides many attempts and modifications to improve this [50-52], the obtained STH efficiencies are still far from this limit in hematite-based photoanodes. The reason for that is the poor electrical properties of the $\alpha-\mathrm{Fe}_{2} \mathrm{O}_{3}$ semiconductor. The hole diffusion length for this semiconductor is as short as $20 \mathrm{~nm}$, while the penetration depth of light is a couple of times larger than this $[52,53]$. Therefore, most of the light absorbed inside the bulk of the medium ( $>20 \mathrm{~nm}$ away from the surface) cannot be utilized for the water oxidation process. In other words, the electron-hole pairs generated inside the bulk recombine before the holes reach to the semiconductorelectrolyte interface. Therefore, nanostructuring is crucial for making hematite photoanodes efficient. Hematite nanostructures, such as nanoparticles, nanowires, nanotubes, and nanocones, are examples of these trapping high surface area morphologies [54-66]. In these three-dimensional scaffolds, light would be trapped and wholly harvested, and the high surface area will increase the probability of hole participation in water oxidation reaction. However, at the meanwhile, many bulk and surface recombination pathways, stemming from the bulky nature of these 3D architectures, can limit the overall water-splitting efficiency of the cell. Moreover, thinning down these nanostructures to radii as small as $20 \mathrm{~nm}$ is a synthetic challenge. The synthesis of ultrathin nanosheets with lateral dimensions as thin as $\sim 10 \mathrm{~nm}$ could be an option to minimize the path length of the holes. However, these nanosheets have also high recombination pathways, and they are not able to harvest the light as efficiently as other 3D [67-71]. Therefore, to approach the maximum STH efficiency, the ideal case is to use a trapping mechanism to fully harvest the light in a thin hematite layer to keep the electron and hole transport path below their diffusion length.

One of the most studied strategies to harvest the light in $\alpha$ $\mathrm{Fe}_{2} \mathrm{O}_{3}$ dimensions as thin as $20 \mathrm{~nm}$ is to integrate these ultrathin layers with impedance-matched trapping 3D photonic scaffold [72-74]. It was theoretically found that a $20-\mathrm{nm} \alpha-$ $\mathrm{Fe}_{2} \mathrm{O}_{3}$ on indium-doped tin oxide (ITO) nanocones can reach a maximum photocurrent value of $12.5 \mathrm{~mA} \mathrm{~cm}{ }^{-2}$ due to the formation of slow waves in gradual impedance matching the condition of nanocones [72]. This value has been calculated by assuming that all the holes will contribute in the watersplitting reaction. However, the drawback of this design is the fact that the real synthesis of these high aspect ratio ITO nanocones is not a simple task. Moreover, a portion of light will pass through the design (considering the fact that ITO is an optically transparent medium and hematite is ultrathin) without getting absorbed by the $\alpha-\mathrm{Fe}_{2} \mathrm{O}_{3}$ in which this deficiency can be quite significant in shorter nanocones. This was improved in later studies, where the authors used a back metallic reflector on the 3D scaffold design [73, 74]. They coated $\mathrm{Al}_{2} \mathrm{O}_{3} 3 \mathrm{D}$ nanocone substrate with a three-layer design made of Ti/Pt-FTO $-\alpha-\mathrm{Fe}_{2} \mathrm{O}_{3}$ and experimentally demonstrated a photocurrent as high as $3.05 \mathrm{~mA} \mathrm{~cm}{ }^{-2}$ at $1.23 \mathrm{~V}$ vs RHE. As can be clearly seen, this value is well below the theoretical limit of $12.5 \mathrm{~mA} \mathrm{~cm}$, while the structure is supposed to perfectly harvest the light. The reason is the high lossy nature of metals (Ti and Pt) compared to low loss $\alpha-\mathrm{Fe}_{2} \mathrm{O}_{3}$. In a nanocone design, light is strongly confined between nanocones, and both metal and semiconductor layers have absorption coefficient to absorb this light. However, metals have much larger extinction compared to hematite, and therefore, most of the light is dissipated in the metal layer, and $\alpha-\mathrm{Fe}_{2} \mathrm{O}_{3}$ cannot fully utilize the incoming broadband solar irradiation. Therefore, it would be better to provide this trapping mechanism in a planar configuration and concentrate the light absorption in the vicinity of the semiconductor layer. The excitation of surface plasmon resonances (SPRs) in noble metal nanostructures is one of these ways [75-79]. The use of gold nanopillars and nanoholes has been proposed to enhance the water-splitting efficiency of the hematite layer [77]. However, a part of the diffracted wave from these plasmonic designs will be harvested by the gold layer that restricts achieving maximum light absorption by the hematite layer. Previously, the use of a simple perfect mirror-semiconductor configuration was proposed by authors where this multilayer mirror provided light perfect absorption in a broad frequency range in the hematite layer [80]. Putting this planar multilayer structure in a V-shaped cell leads to a photocurrent value of $4 \mathrm{~mA} \mathrm{~cm}^{-2}$ in a design made of 26-nm-thick Ti-doped $\alpha-\mathrm{Fe}_{2} \mathrm{O}_{3}$ films. Besides all these ideas, the utilization of Mie resonances in a hematite nanobeam was demonstrated as an efficient photoanode in light-driven water-splitting cell [81].

In this work, we propose an elegant water-splitting photoanode design comprising metal-oxide-semiconductorsemiconductor (nanograting/nanopatch) $\left(\mathrm{MOSS}_{\mathrm{g} / \mathrm{p}}\right)$ as a highly efficient trapping configuration to harvest the light in a broad frequency range while keeping the active layer dimensions much smaller than the incident light wavelength. In this architecture, the metal layer is an optically thick aluminum (Al) mirror, the oxide layer is an ultrathin ITO layer, the planar semiconductor layer is a 16-nm-thick $\alpha-\mathrm{Fe}_{2} \mathrm{O}_{3}$ layer, and the top nanostructured semiconductor is made of ultrathin cadmium sulfide $(\mathrm{CdS})$. Here, the absorption of the light in shorter wavelengths is supported by the efficient coupling of the incident light into metal-oxide-semiconductor (MOS) cavity modes, while the longer wavelengths are harvested by the 
excitation of Mie resonances supported by $\mathrm{CdS}$ grating/patch. Therefore, by the superposition of these two responses, an efficient light harvesting can be achieved with an absorption above 0.8 in most of the spectral region above the optical band gap of hematite. The absorption of the light in dimensions below the diffusion length of $\alpha-\mathrm{Fe}_{2} \mathrm{O}_{3}$ will increase the collection probability of the photogenerated carriers. Moreover, the proper band alignment between $\mathrm{CdS}$ and $\alpha-\mathrm{Fe}_{2} \mathrm{O}_{3}$ will facilitate charge separation and reduce their recombination probability, and this in turn will maximize hole participation in a water-splitting reaction. Our theoretical calculations prove that the use of this semiconductor-based metamaterial can provide a photocurrent as high as $10.7 \mathrm{~mA} \mathrm{~cm}^{-2}$ and that is quite close to the maximum theoretical value. Similar to other theoretical studies, this value has been obtained by assuming that all of the generated carriers are collected due to the fact that the proposed design has dimensions smaller than the diffusion length of $\alpha-\mathrm{Fe}_{2} \mathrm{O}_{3}$ and the existence of an $\alpha-\mathrm{Fe}_{2} \mathrm{O}_{3}$ $\mathrm{CdS}$ interface will further enhance the carrier separation probability. Our simulations also demonstrate that the proposed nanopatch design has absolute polarization insensitivity and good angular response and these are important for practical applications. Furthermore, compared to other trapping schemes, this structure has a very simple design configuration and can be easily scaled to large scales using common fabrication tools such as nanoimprint lithography. This study shows that a proper ultrathin planar and large-scale compatible design configuration can offer excellent optical and electrical properties in which a highly efficient photoelectrochemical water-splitting cell can be made using common and highly repeatable nanofabrication technologies.

\section{Results and Discussion}

One of the most intensively explored semiconductor-based metamaterial designs is the metal-semiconductor (MS) configuration. The strong interface effects, induced by a bottom mirror, can provide light nearly perfect absorption in this design. In this configuration, the right choice of semiconductor geometries can significantly enhance the light-matter interaction [82-87]. However, this configuration can enhance light absorption to some extent. In the other words, this design has its own limit. To scrutinize the limits of this semiconductorbased MS metamaterial structure, we have adopted a modeling approach based on the transfer matrix method (TMM) to find the ideal condition for light perfect absorption in a visible (Vis) portion of the solar spectrum. The studied design architecture is shown in Fig. 1a. In this model, the bottom metal is chosen as highly reflecting silver (Ag) metal [88]. Afterward, the ideal real and imaginary parts of permittivity have been extracted for four different semiconductor thicknesses of $D_{S}=5,15,25$, and $35 \mathrm{~nm}$. For this aim, the reflection of the design is calculated using TMM. In this modeling, we assume that the MS structure is bounded with a material of $\varepsilon_{\mathrm{A}}$ which is air. For transverse magnetic (TM) polarized light, taking the $\mathrm{H}_{\mathrm{y}}$ as [89]:

$H_{y}(z)=\left\{\begin{array}{l}A_{i} e^{i k_{A}\left(z-D_{S}\right)}+A_{r} e^{-i k_{A}\left(z-D_{S}\right)}, z>D_{S} \\ S_{11} e^{i k_{S} z}+S_{12} e^{-i k_{S} z}, 0<z<D_{S} \\ M_{21} e^{i k_{M} z}+M_{22} e^{-i k_{M} z},-D_{M}<z<0\end{array}\right\}$

and applying the appropriate boundary conditions, the reflection of the incident light from the structure can be obtained as $R=\left|F_{12} / F_{11}\right|^{2}$. Here, $F=\left[\begin{array}{c}F_{11} \\ F_{12}\end{array}\right]=a^{-1} s_{11} s_{12}^{-1} m_{21} m_{22}^{-1}$ where:

$a=\left[\begin{array}{cc}1 & 1 \\ i k_{A} / \varepsilon_{A} & -i k_{A} / \varepsilon_{A}\end{array}\right]$,

$$
\begin{aligned}
s_{11} & =\left[\begin{array}{cc}
1 & 1 \\
i k_{S} / \varepsilon_{S} & -i k_{S} / \varepsilon_{S}
\end{array}\right], s_{12} \\
& =\left[\begin{array}{cc}
e^{i k_{S} D_{S}} & e^{-i k_{S} D_{S}} \\
i k_{S} e^{i k_{S} D_{S}} / \varepsilon_{S} & -i k_{S} e^{-i k_{S} D_{S}} / \varepsilon_{S}
\end{array}\right], \\
m_{21} & =\left[\begin{array}{cc}
1 & 1 \\
i k_{M} / \varepsilon_{M} & -i k_{M} / \varepsilon_{M}
\end{array}\right], m_{22} \\
& =\left[\begin{array}{cc}
e^{i k_{M} D_{M}} & e^{-i k_{M} D_{M}} \\
i k_{M} e^{i k_{M} D_{M}} / \varepsilon_{M} & -i k_{M} e^{-i k_{M} D_{M}} / \varepsilon_{M}
\end{array}\right],
\end{aligned}
$$

and $k_{i=(A, S, M)}=\sqrt{\varepsilon_{i} \omega^{2} / c^{2}-k_{x}^{2}}$ in which $c$ is the speed of light, $D_{S}$ is the semiconductor layer thickness, and $D_{M}$ is the bottom metal layer thickness. Using the above-mentioned formulations, the real and imaginary parts of ideal material permittivity have been calculated. These regions have been exhibited as highlighted regions. It should be mentioned that the ideal region is defined as the range of permittivity values in which an absorption above 0.8 (reflection below 0.2 ) can be attained. Therefore, if the real and imaginary parts of the permittivity of a semiconductor are located inside these regions, we can say that the MS design has harvested above $80 \%$ of the incident light in that specific wavelength value. The matching of these ideal regions has been compared with those of the permittivity data of three different semiconductors, including germanium (Ge, as a narrow-band-gap semiconductor), perovskite (PVSK, as an organic narrow-band-gap semiconductor), and $\mathrm{Fe}_{2} \mathrm{O}_{3}$ (hematite, as a narrow-band-gap metal oxide). As shown in Fig. 1b, the real part of permittivity stays out of the ideal region for all three types of semiconductors and that proves the poor absorption characteristic of the MS structure in such thin layers. As we go toward larger thicknesses, the ideal region moves toward smaller positive values, and, therefore, matching can be met in a narrow frequency range. As shown in Fig. 1c, this matching is obtained in a range of 600 

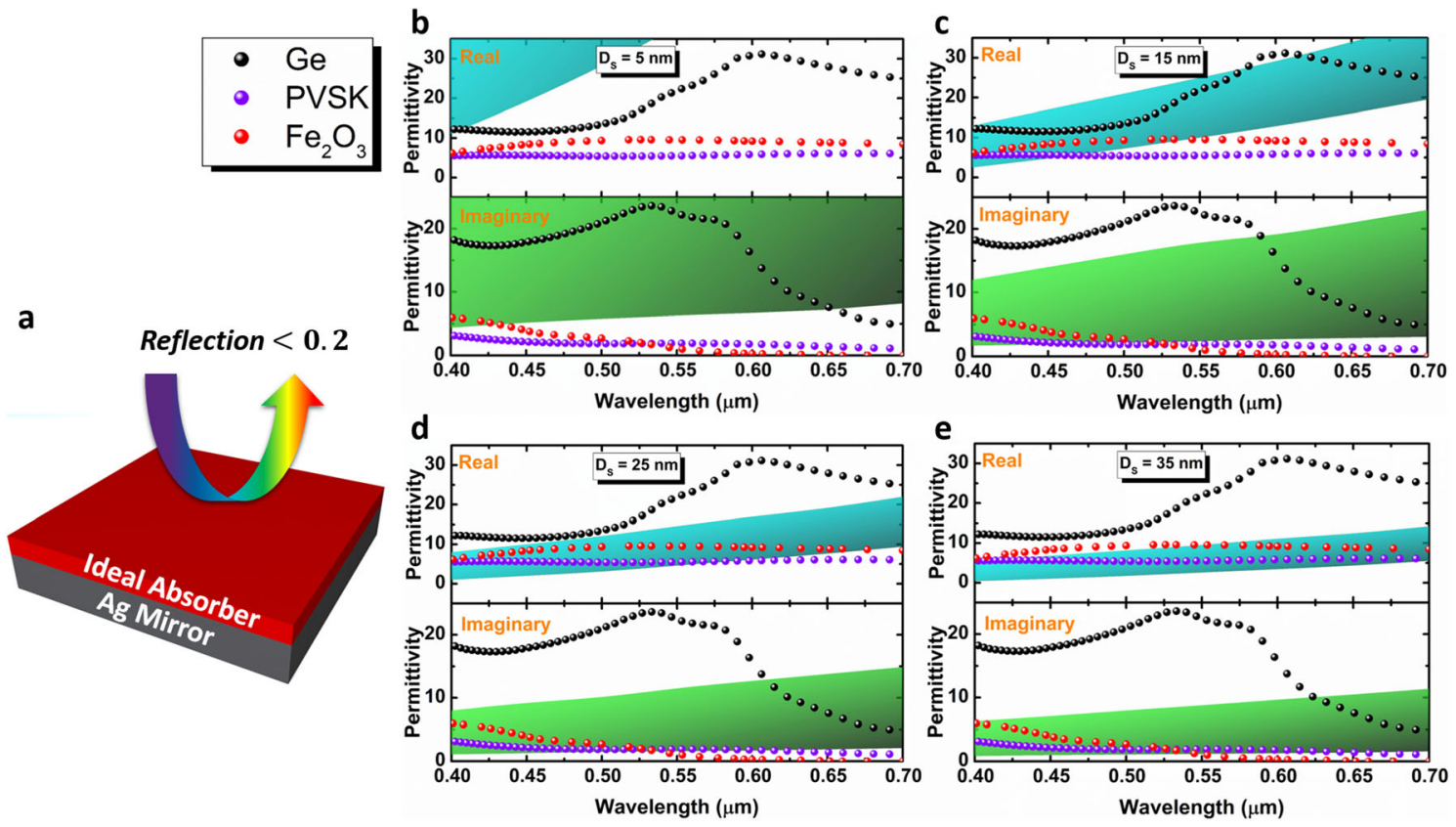

Fig. 1 (a) Schematic representation of the studied MS design to find the ideal condition for light perfect absorption. The comparison between the ideal region and permittivity data of different semiconductors for four different $D_{\mathrm{S}}$ values of (b) 5, (c) 15, (d) 25, and (d) $35 \mathrm{~nm}$

$700 \mathrm{~nm}$ for Ge (in agreement with previous studies [85-87]), while the matching is satisfied in wavelength values below $500 \mathrm{~nm}$ for PVSK and $\mathrm{Fe}_{2} \mathrm{O}_{3}$ [82]. Increasing $D_{S}$ to $25 \mathrm{~nm}$, Ge loses its matching, while $\mathrm{Fe}_{2} \mathrm{O}_{3}$ absorption upper edge experiences a red shift (see Fig. 1d). The thicker layers, however, make the ideal region narrower, and the matching is lost for $\mathrm{Ge}$ and $\mathrm{Fe}_{2} \mathrm{O}_{3}$. Therefore, the optimal thickness for Ge is $15 \mathrm{~nm}$, while the optimal condition for $\mathrm{Fe}_{2} \mathrm{O}_{3}$ is attained in a thickness of $25 \mathrm{~nm}$ where an absorption above 0.8 can be achieved from 400 to $525 \mathrm{~nm}$. For the case of PVSK, the best results are recorded in the semiconductor layer thickness of $35 \mathrm{~nm}$ in which the absorption bandwidth (BW) can cover almost the whole Vis regime (400-650 nm). Therefore, in an MS configuration, every material has its own ideal semiconductor layer thickness. However, this absorption response is limited to a specific spectral range, and further improvement can be obtained by tailoring the design architecture.

Gaining an insight on the limits of this configuration, in the next step, numerical simulations are conducted to find the optimum design geometries for a highly efficient $\mathrm{Fe}_{2} \mathrm{O}_{3}$-based watersplitting photoanode. For this goal, a commercial finitedifference time-domain (FDTD) software package (Lumerical FDTD Solutions) [90] is exploited to numerically simulate the design optical performance. The structure is excited with a broadband plane wave spanning from 400 to $750 \mathrm{~nm}$ in our desired polarization and incidence angles. The simulation is performed in two-dimensional (2D) mode. The boundary conditions (BCs) in the $x$ and $y$ directions are chosen as periodic. The bottom and top $\mathrm{BCs}$ are set as a perfectly matched layer (PML). Since the bottom layer is an optically thick mirror with almost no transmission, it can be safely said that there is zero transmittance in this device. Therefore, the absorption spectra can be calculated directly from the reflection spectra using the following formula $A=1-R$. The reflection monitor is placed on top of the incident source excitation plane to only collect the reflected wave. Since this structure is going to be used in a water-splitting setup, we need to consider the electrical properties of the design as well. Due to the large energy level difference between the Fermi level of Ag and conduction band of $\mathrm{Fe}_{2} \mathrm{O}_{3}$, this structure may suffer from poor charge collection efficiency. Therefore, a conductive transparent layer should be embedded between these two layers to facilitate electron transfer. Optical transparent conductive indium-doped tin oxide (ITO) is an excellent option that can provide the proper conditions for the optical and electrical performance of the design. To gain insight on the impact of the ITO layer in the absorption functionality of the system, the response of the MS structure is compared with that of the metal-oxidesemiconductor (MOS) cavity where the oxide is the ITO layer with different thicknesses. Figure $2 \mathrm{a}$ shows the absorption spectra of the MS structure for different semiconductor thicknesses $\left(D_{S}\right)$ spanning from 5 to $25 \mathrm{~nm}$ with a step size of $5 \mathrm{~nm}$. The same sweep is performed for an MOS junction with different oxide thicknesses of 5,10,15, and $20 \mathrm{~nm}$, as shown in Fig. $2 \mathrm{~b}-\mathrm{e}$. To have a better qualitative comparison, the average visible light absorption (400-700 nm) has been calculated using the following formula:

Average Absorption $=\frac{\int_{400 \mathrm{~nm}}^{700 \mathrm{~nm}} \alpha(\lambda) \Delta \lambda}{\int_{400 \mathrm{~nm}}^{700 \mathrm{~nm}} \Delta \lambda}$ 

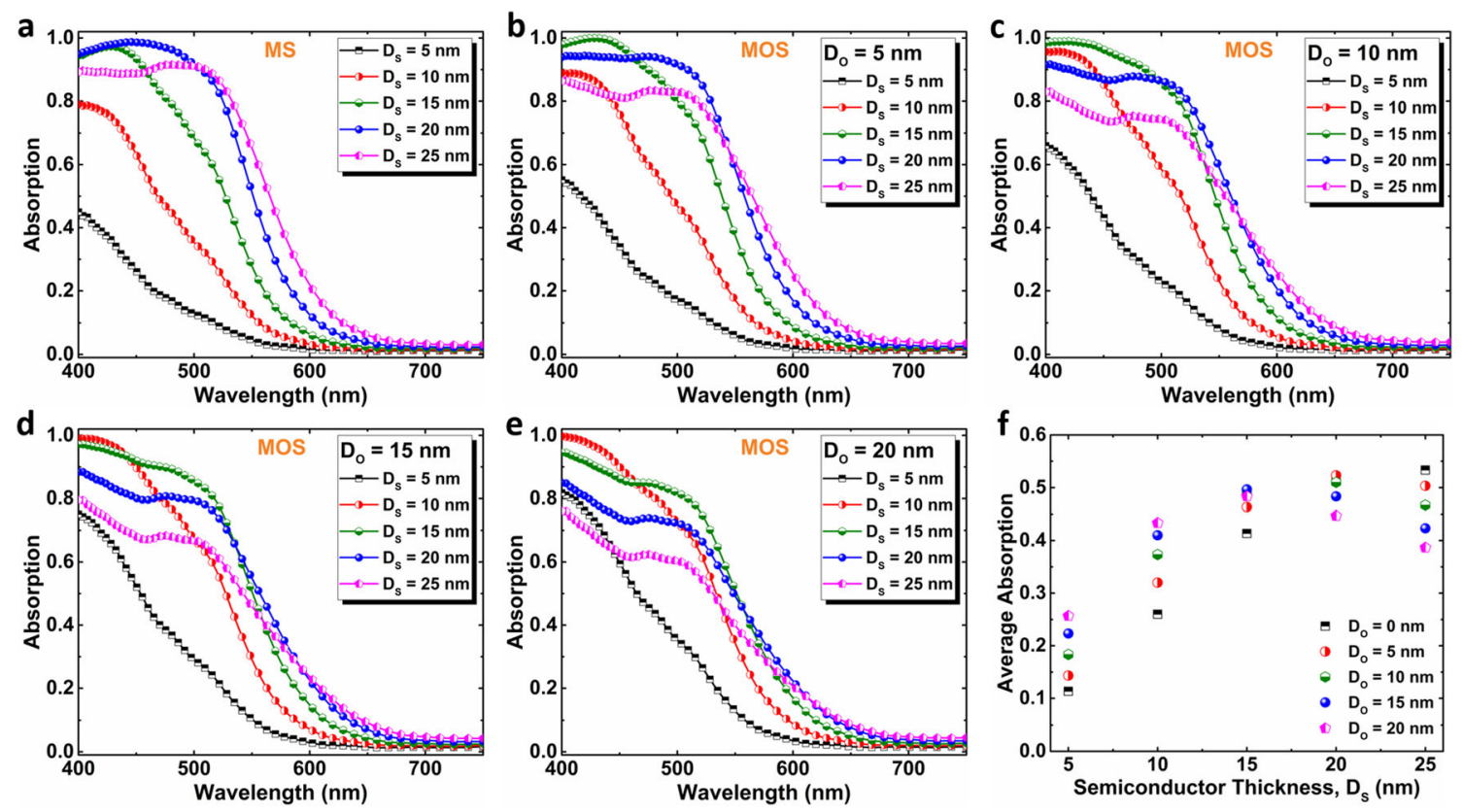

Fig. 2 The absorption spectra for (a) MS design with different semiconductor layer thicknesses. The absorption spectra for MOS designs with different semiconductor layer thicknesses for oxide layer thicknesses of (b) 5, (c) 10, (d) 15, and (e) $20 \mathrm{~nm}$. (f) The calculated average absorption for different MOS designs where $\alpha$ is absorption coefficient and $\lambda$ is the incident light wavelength. Looking at Fig. $2 \mathrm{f}$, it can be seen that the highest average absorption for an MS structure is attained at the $D_{S}$ value of $25 \mathrm{~nm}$ that is in line with our findings from previous section modeling. In general, by moving to an MOS configuration, the absorption decreases compared to that of an MS junction. However, in the $D_{S}=20 \mathrm{~nm}$, the oxide thickness of $5 \mathrm{~nm}$ leads to a maximum average absorption of 0.53 which is close to that of the maximum amount for an MS junction. Therefore, by embedding an ultrathin ITO layer, not only the optical absorption stays high but also the electrical charge separation for photoinduced electrons will be facilitated compared to an MS design. In this optimal condition, above 0.8 absorption is acquired from 400 to $525 \mathrm{~nm}$, which is in agreement with our expectations from the limit of this design. Considering the optical band gap of $\mathrm{Fe}_{2} \mathrm{O}_{3}$ which extends up to $590 \mathrm{~nm}$, the portion of the light in longer visible range cannot be harvested efficiently, and thus the photoactivity of this photoanode is restricted in this planar configuration. Thus, we need to exploit a new design configuration to be able to extend the absorption edge toward the absorption edge of $\mathrm{Fe}_{2} \mathrm{O}_{3}$. This could be attained by adding Mie resonances into the design. It is in this way that the absorption in shorter wavelengths will be supported by the cavity design and the longer $\lambda \mathrm{s}$ will be harvested by the help of Mie resonances. To achieve this goal, we proposed the design configuration as shown in Fig. 3a. As shown in this panel, cadmium sulfide (CdS) grating has been added into the top of the MOS junction. CdS is a large band gap semiconductor, and it is expected that it would not hamper the cavity response of the underlying MOS design. Another reason why we used this material as a grating layer is its excellent band alignment matching with $\mathrm{Fe}_{2} \mathrm{O}_{3}$, in which photoinduced electron-hole pairs inside the hematite layer will be spatially isolated by $\mathrm{CdS}$ and consequently the recombination probability of these carriers will significantly decrease [91, 92]. The geometries of each layer have been exhibited in the same panel. In this architecture, $P$ is the periodicity of the grating, $W$ is the width of the grating which is an $\alpha$ portion of $P, D_{G}$ is the grating thickness, and $D_{S}$ is $\mathrm{Fe}_{2} \mathrm{O}_{3}$ layer thickness. The ITO layer is fixed at an optimal thickness of $5 \mathrm{~nm}$. To begin with, we need to know the optimum thickness of the grating layer. For this, a sweep is conducted on the $D_{G}$ values from 5 to $25 \mathrm{~nm}$ with a step size of $5 \mathrm{~nm}$. These sweeps are repeated for different semiconductor layer thicknesses of $10,15,20$, and $25 \mathrm{~nm}$. In this set of simulations, the periodicity is fixed at $250 \mathrm{~nm}$, and $\alpha$ is set at 0.5 . As shown in Fig. 3b-e, a new peak is added to the response of an MOS design that originated from the existence of CdS grating. This peak gets a slight redshift as the $D_{G}$ increases. Moreover, as already explained, independent from the grating geometries, the short wavelength absorption response is only defined with the MOS cavity dimensions. A proper design should keep the absorption response above 0.8 in a wide frequency range, and this can be obtained by adjusting the spectral position of the Mie resonance related peak in the vicinity of the absorption upper edge of MOS design. This has been achieved in the design geometries of $D_{s}=15 \mathrm{~nm}$ and $D_{G}=25 \mathrm{~nm}$ in which absorption has been retained above 0.8 up to $550 \mathrm{~nm}$.

Based on the guided-mode resonance theory, the spectral position of these Mie resonances are not only tuned with core layer thickness, but also the spacing between gratings can alter this resonance [81]. The optimal mode coupling conditions is 

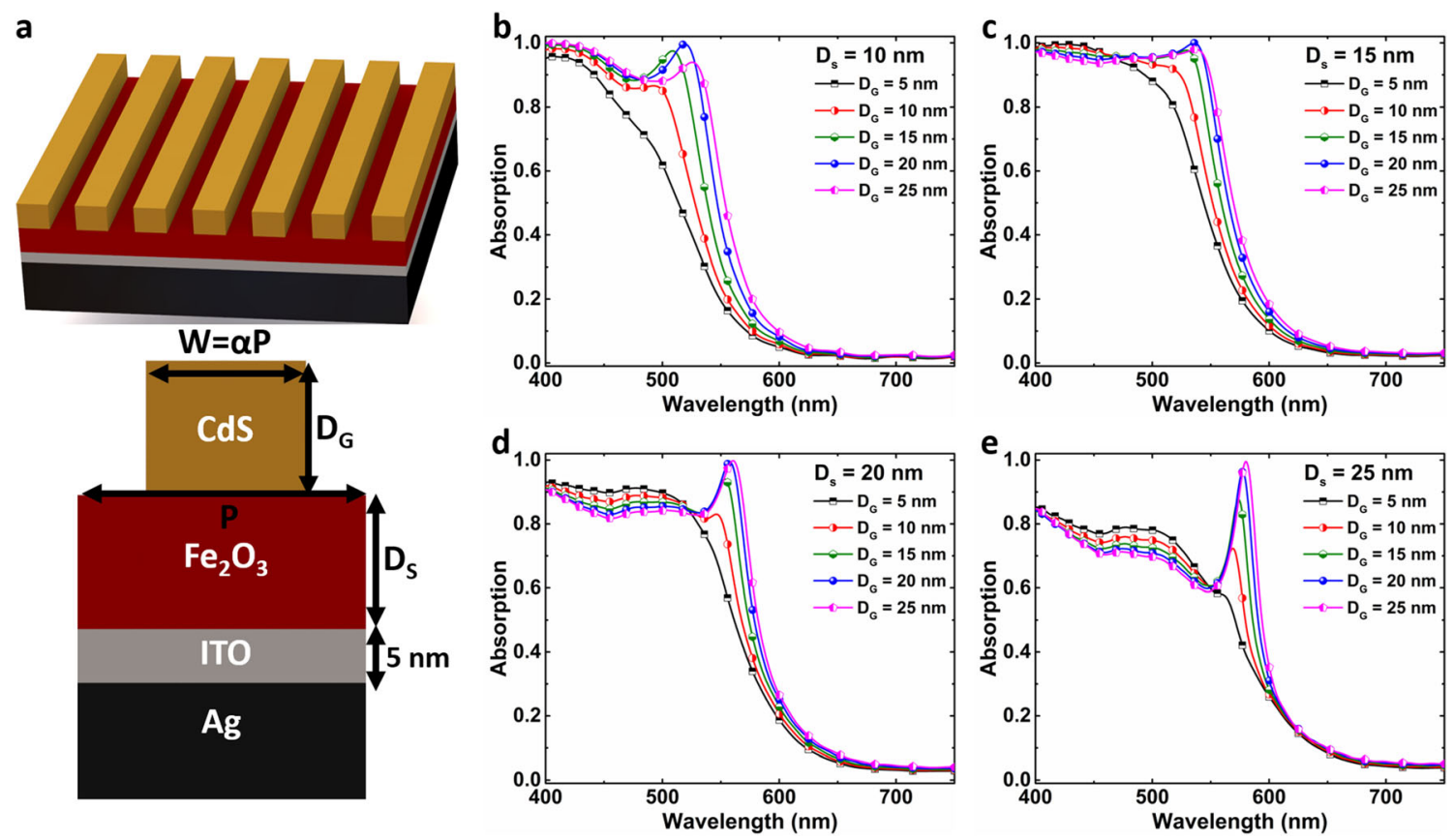

Fig. 3 (a) Schematic representation of MOSS $_{\mathrm{g} / \mathrm{p}}$ design showing its different layers and geometries. The absorption spectra with different $\mathrm{D}_{\mathrm{G}}$ values for four different $\mathrm{D}_{\mathrm{S}}$ values of (b) 10 , (c) 15 , (d) 20 , and (e) $25 \mathrm{~nm}$

achieved when the momentum matching condition $(G=k)$ is satisfied. In this formula, $k=2 \pi \mathrm{n}_{\text {eff }} / \lambda$ where $\mathrm{n}_{\text {eff }}$ is the effective mode index for the quasi-guided mode supported by the design. To gain an understanding on the physics behind these absorption peaks, we have plotted the ratio of the absorbed power in this grating design to that of the best planar design as a function of $G$. To find the total absorbed power inside the semiconductor layer, we have employed the following formula:

$A(\lambda)=\frac{1}{P_{\text {incident }}} \int_{400 \mathrm{~nm}}^{700 \mathrm{~mm}} 0.5 \omega \operatorname{imag}\left(\varepsilon_{\text {Fe2O3 }}\right)|E(x, z, \lambda)|^{2} d x d z$

To have a better visualization, the bottom limit of this counter plot is set at one. As Fig. 4a illustrates, increasing the periodicity (reducing the $\mathrm{G}$ parameter) of the structure increases the strength of the ratio where an enhancement as high as 3.5 can be achieved compared to the optimal planar design. Moreover, together with this enhancement, a redshift is experienced as we increase the spacing of the grating. Considering the previous results, although the enhancement ratio increases with increasing the periodicity, the absolute absorption of the design exponentially drops in a longer wavelength. Therefore, we also need to examine the absolute absorption values of the grating structure as the function of $P$. This data has been plotted in Fig. 4b. As this graph clearly illustrates, as we go toward larger grating spacing, a dip starts to appear in wavelengths between 520 and $580 \mathrm{~nm}$. In fact, increasing the periodicity decouples the absorption response due to cavity modes and those due to Mie resonances. Thus, in our case, a moderate $P$ will lead to a maximum absorption response. To finalize our simulations, we pick three periodicities of 250,290 , and $330 \mathrm{~nm}$ and span $\alpha$ (corresponding to the width of grating) from 0.3 to 0.7. According to the results presented in Fig. $4 \mathrm{c}-$ $\mathrm{e}$, the optimal condition is realized when the periodicity is set at $290 \mathrm{~nm}$, and $\alpha$ is 0.4 . In this condition, the absorption BW (defined as the spectrum with absorption above 0.8 ) is expanded from 400 to $565 \mathrm{~nm}$. To the best of our knowledge, this is the broadest absorption BW reported for a planar subwavelength hematite-based photoanode.

To have a solid understanding on the nature of these resonance modes, we have simulated the electric field (E-field) and magnetic field (H-field) distributions within the cavity in two wavelengths. One is located at $450 \mathrm{~nm}$ that corresponds to the place that we have near unity flat absorption and the other is chosen as $555 \mathrm{~nm}$ that is the spectral position of the second resonance peak. Figures 5a depicts the E-field and $\mathrm{H}$-field distributions at $\lambda=450 \mathrm{~nm}$. As this figure implies, the Efield is mainly concentrated in the walls of CdS grating, and magnetic field has been dominantly focused beneath of the grating. The formation of the standing wave just below the grating has localized the incoming incident light within the cavity where this light is mainly harvested with the $\mathrm{Fe}_{2} \mathrm{O}_{3}$ semiconductor layer due to its higher extinction coefficient. However, these profiles are significantly different for the second resonance peak. In this case, as illustrated in Fig. 5b, the E-field is tightly confined within the low refractive index ITO layer which is sandwiched with two high-index mediums. This distribution again confirms our above explanations that second resonance mode is originated from the coupling of 
a

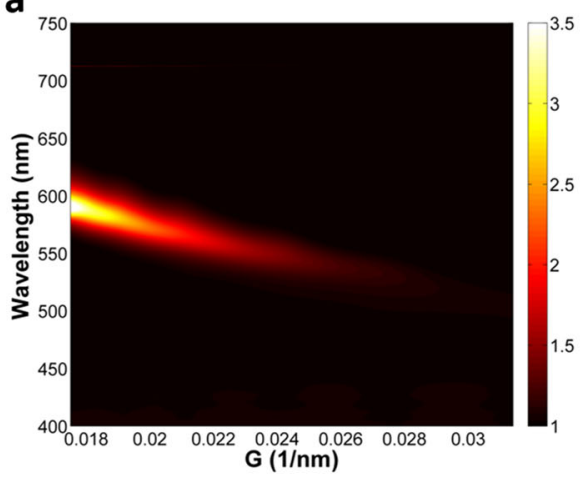

b

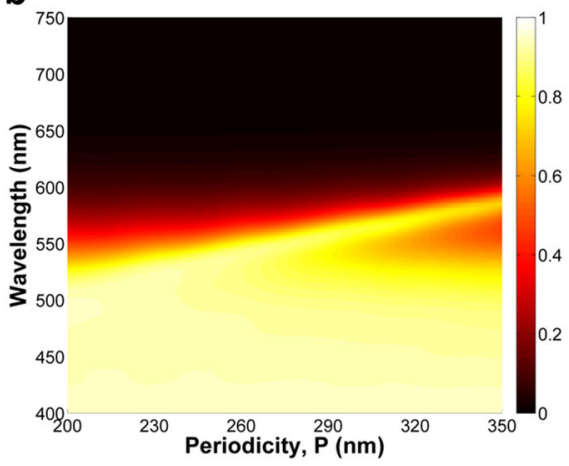

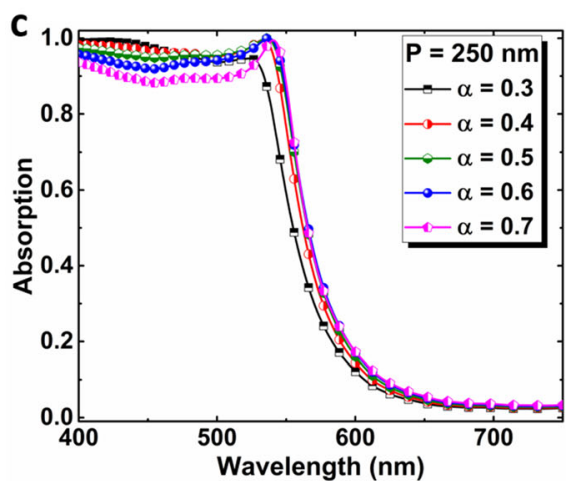
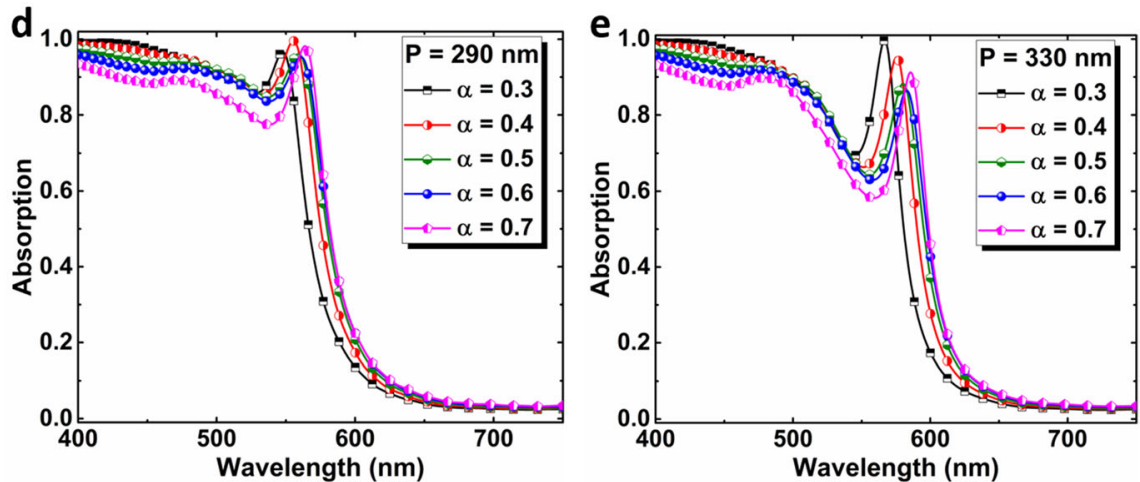

Fig. 4 (a) The ratio of the absorbed power in the grating design to that of the best planar design as a function of $G$. (b) The absolute absorption response of the grating design as a function of $P$. The absorption spectra of the design as a function of $\alpha$ for different $P$ values of (c) 250 , (d) 290 , and (e) $330 \mathrm{~nm}$

incident light into guided mode resonances, supported with the cavity design. Moreover, $\mathrm{H}$-field, in this case, is dominantly localized between the nanogratings rather than being confined underneath the grating.

Taking all the above-mentioned results into consideration, the proposed design architecture can efficiently harvest the light in a broad frequency range through the superposition of MOS cavity modes and grating based-guided mode resonances. However, the only drawback with this design is its polarization sensitivity of the grating. As we move from TM polarization toward transverse electric (TE) polarization, the excitation of guided mode resonances is hampered. To mitigate this drawback, we replaced the grating with a square nanopatch. The same optimization process is applied to this design to maximize its absorption BW. The optimal geometries for this case were found as $D_{O}=5 \mathrm{~nm}, D_{S}=16 \mathrm{~nm}, D_{G}=$ $25 \mathrm{~nm}, P=300 \mathrm{~nm}$, and $\alpha=0.55$. The optimized results for all three cases of planar, grating, and patch have been plotted in Fig. 6b. As this figure clearly shows, relatively similar responses have been attained for both grating and patch designs. Figure $6 \mathrm{c}-\mathrm{d}$ plot the absorption spectra of grating and patch designs, respectively. The bottom limit of the contour plot is set at 0.8 to provide a better visualization of the narrowing of the absorption BW. According to these plots, for the gratingbased metasurface design, changing the polarization angle from $0^{\circ}$ to $90^{\circ}$ imposes a blue shift in the upper absorption a

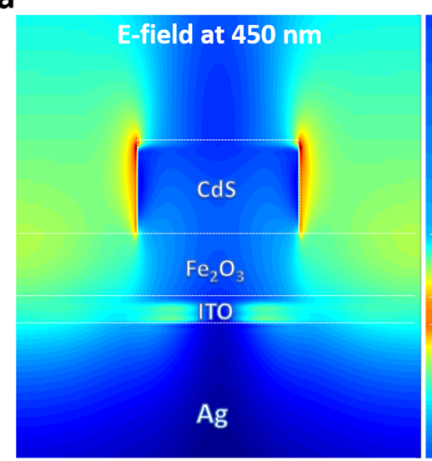

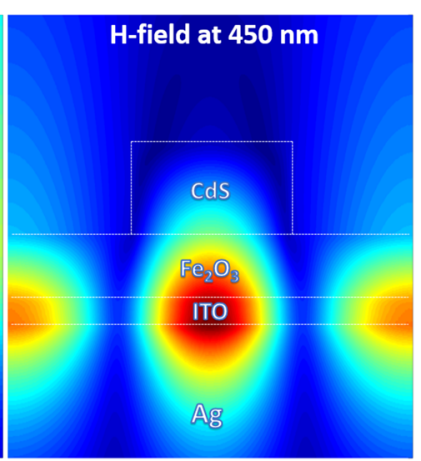

b
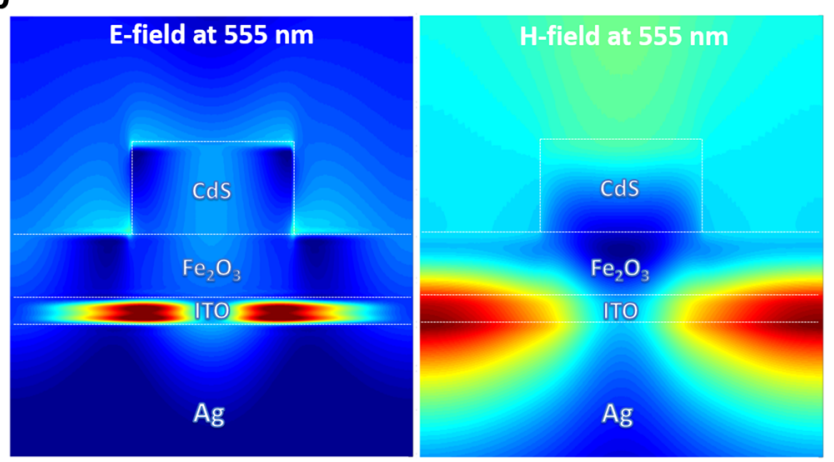

Fig. 5 The E-field and H-field distribution for the proposed metasurface design in two different wavelengths of (a) 450 and (b) $555 \mathrm{~nm}$ 

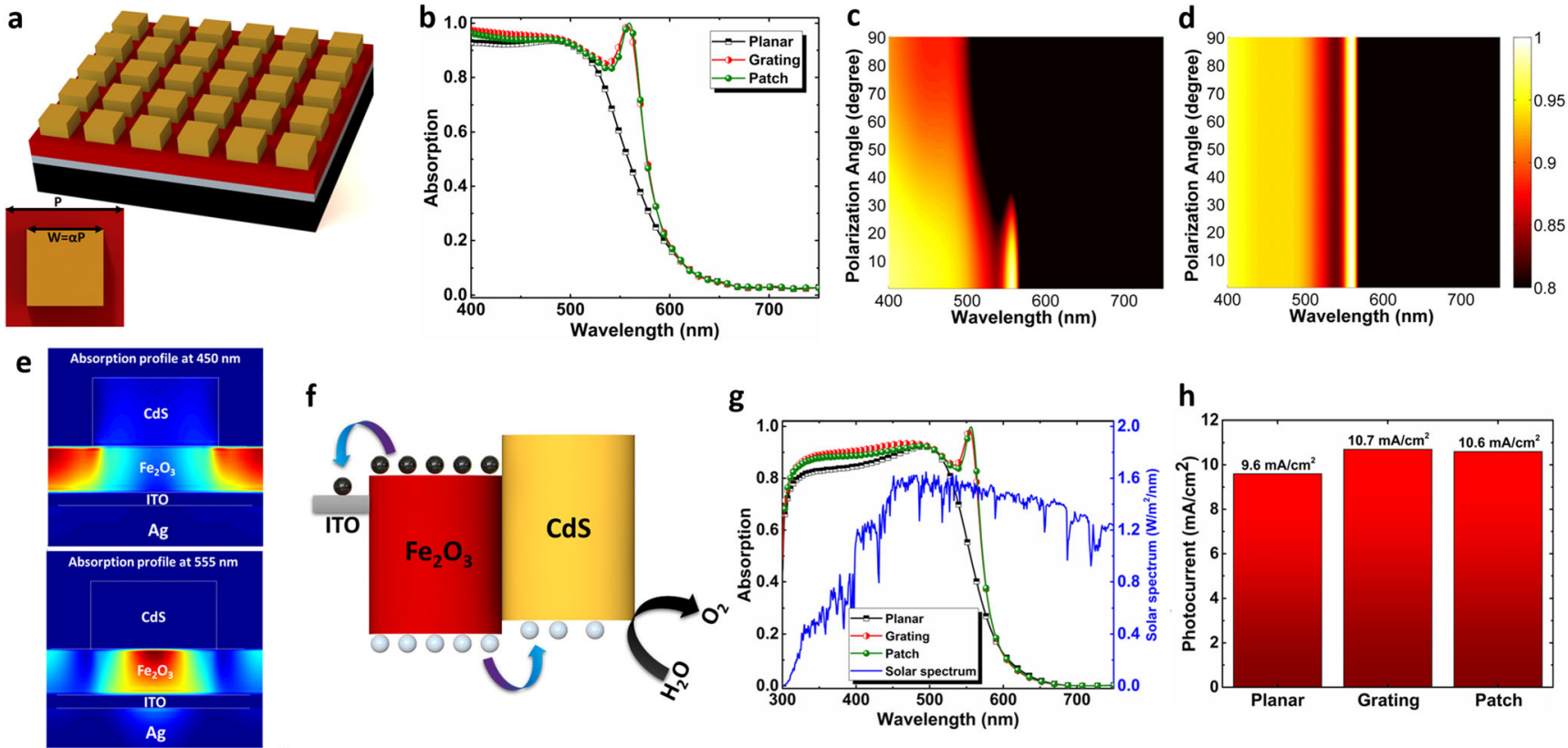

f

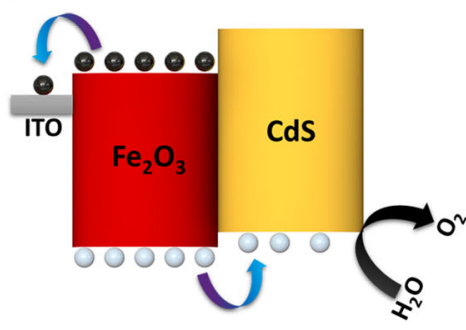

g
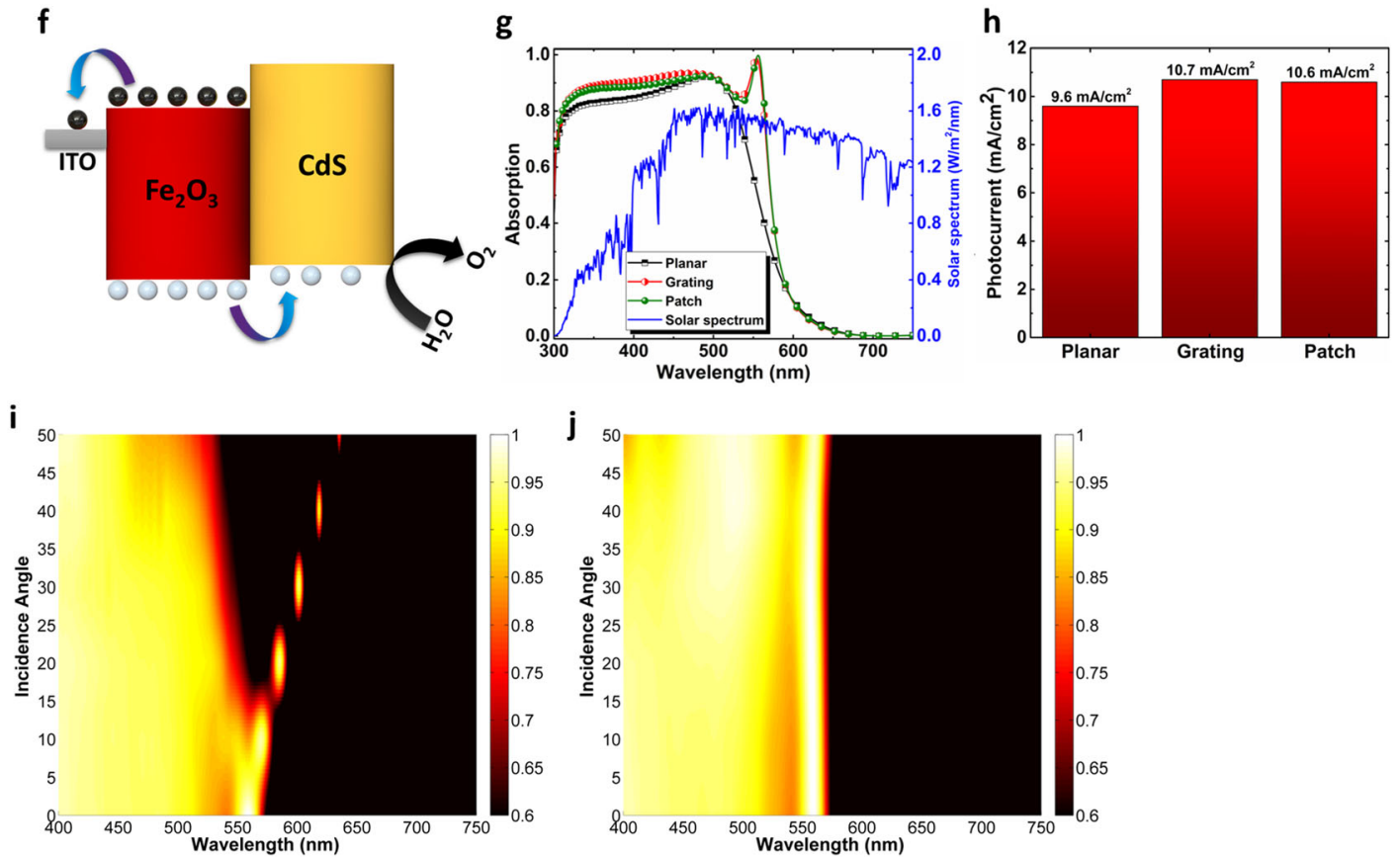

Fig. 6 (a) The schematic representation of the proposed metasurface design with a nanopatch top layer. (b) The comparison of the absorption spectra for three different design architectures. The absorption spectra of the (c) grating and (d) patch as a function of the polarization angle. (e) The absorption profile across the design in two different wavelengths of 450 and $555 \mathrm{~nm}$. (f) The band alignment for
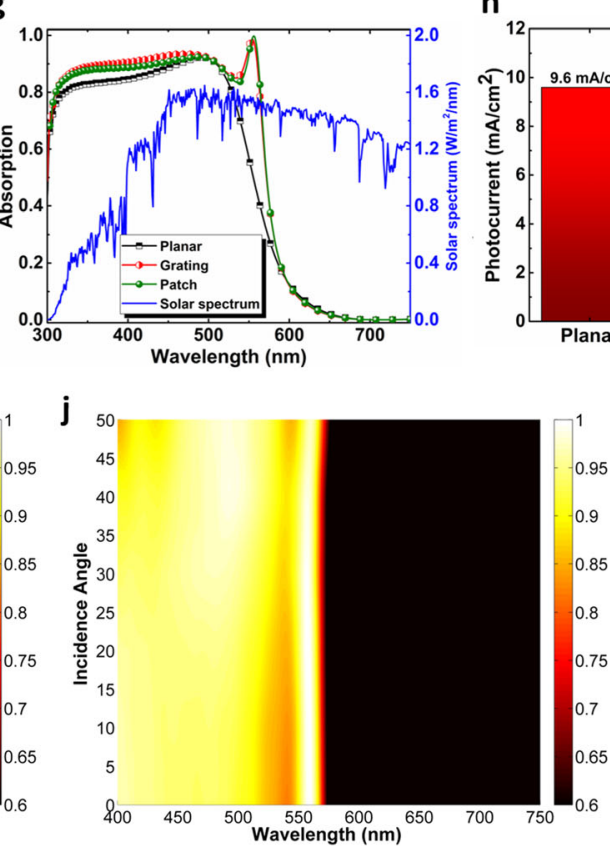

the proposed design. (g) Calculated absorption spectra for three different proposed designs and the solar irradiation spectrum in a range of 300-750 nm. (h) The calculated photocurrent values. The angular absorption response of the patch-based metasurface design for (i) TM and (j) TE polarizations

edge from 565 to $500 \mathrm{~nm}$. Because incoming solar irradiation is an unpolarized light, this could limit the practical use of this structure for large-scale applications. Looking at polarization dependency of absorption profile for patch design, we can see that an absolute polarization-insensitive design has been obtained in this case. The absorption profile in a view plane cutting through the middle of the patch has been calculated using Eq. 4 and has been plotted in Fig. 6e. As this figure clearly implies, most of the light is harvested inside the $\mathrm{Fe}_{2} \mathrm{O}_{3}$ layer. In the shorter wavelength regime, a negligible part of incident power is harvested inside the CdS layer, and in the upper band $\mathrm{Ag}$ mirror, it has a weak absorbing feature. Moreover, the absorption in $\lambda=450 \mathrm{~nm}$ is mainly in the region between two patches. Looking back into the $\mathrm{E}$ - and $\mathrm{H}$-fields distributions for this wavelength, we can see that in the region between two patches, both fields have a moderate intensity, while in the region below the patch, the E-field has a quite

weak amplitude. That is why the light is mainly harvested in the spacing area. Similarly, for the longer wavelength resonance peak, the localization of the E-field in the region underneath the top nanoresonator will lead to the harvesting of the incident light in this area. The near unity light absorption in a thickness of approx. $15 \mathrm{~nm}$ could lead to a maximized collection efficiency. This efficiency could be further substantiated by the selective carrier's separation in $\mathrm{Fe}_{2} \mathrm{O}_{3}-\mathrm{CdS}$ interface. As Fig. $6 \mathrm{f}$ illustrates, upon the excitation of the metasurface design with solar irradiation, electron-hole pairs are generated inside the $\mathrm{Fe}_{2} \mathrm{O}_{3}$ layer. The photoinduced holes are transferred into more energetically favorable valance band of $\mathrm{CdS}$, and then they participate into water oxidation process. The electron conjugate will also move to ITO and from there into Fermi level of $\mathrm{Ag}$ to be collected in the external circuit. To quantitatively compare these three designs, we have calculated the photocurrent as a figure of merit for the photoactivity of 
the photoanode. We assumed that all of the photogenerated electron-hole pairs can contribute to the photocurrent before they recombine, which is a fair assumption when taking the above-mentioned statements into consideration. The photocurrents of these metasurfaces are calculated using:

$$
\left.J\left(m A / \mathrm{cm}^{2}\right)=(e / h c)\right]_{300 \mathrm{~nm}}^{750 \mathrm{~nm}} A(\lambda) I_{A M 1.5} \lambda d \lambda
$$

where $J$ is the photocurrent density, $I_{A M 1.5}$ is the ASTM Air Mass 1.5 solar spectrum, and $A(\lambda)$ is the spectral absorption calculated using Eq. 4. The calculated absorption spectra in a range of 300-750 nm have been exhibited in Fig. 6g. Using this data and the $I_{A M 1.5}$ spectrum, the photocurrent in each of these three cases has been found and plotted in the Fig. $6 \mathrm{~h}$. While the photocurrent is recorded to be $9.6 \mathrm{~mA} / \mathrm{cm}^{2}$ for the planar case, this value has been improved to $10.7 \mathrm{~mA} / \mathrm{cm}^{2}$ and $10.6 \mathrm{~mA} / \mathrm{cm}^{2}$ for grating and patch-guided mode design. These values are quite close to the theoretical maximum photocurrent value of $12.5 \mathrm{~mA} / \mathrm{cm}^{2}$ for a hematite-based photoanode. This has been acquired by the delicate design of the metasurface where the coupling of incident light into both cavity modes and guided mode resonances has been led in highly efficient light harvesting in a broad wavelength range below the band gap of the semiconductor. Finally, the angular response of the system has also been simulated to provide a comprehensive study on the proposed metasurface design. For this aim, the absorption profile of the patch-based cavity has been plotted for two different polarizations of TM and TE, as depicted in Fig. 6i-j. As we can see from these panels, the absorption response for TM polarization gets narrower as we go to wider excitation angles. In the meantime, the response for TE-polarized light stays almost intact in all incident angles. Considering the fact that, in TE polarization, the transverse component of $\mathrm{E}$ field does not change with the light angle of incidence, the Mie resonance excitation is supported in all oblique angles. Therefore, all of the obtained results prove that this $\mathrm{MOSS}_{\mathrm{g} / \mathrm{p}}$ is an efficient design for water-splitting application. Further improvement can be obtained by engineering the semiconductor-semiconductor and semiconductor-electrolyte interfaces using sub-nanometer ultrathin layers $[93,94]$. Such ultrathin layers would not change the optical response of the design, while it significantly improves its electrical behavior.

\section{Conclusion}

In this study, we proposed a design strategy based on semiconductor metasurfaces to maximize the light-matter interaction in dimensions much smaller than the wavelength and close to the hole diffusion length of a semiconductor. For this aim, we first found the limitations of a simple MS design using TMM modeling. Later, the impact of adding a nanostructured semiconductor-based grating on top of an MOS cavity design is explored. Our findings demonstrate that the proposed $\operatorname{MOSS}_{\mathrm{g} / \mathrm{p}}$ design can absorb light in a broad frequency range by the simultaneous excitation of cavity modes and Mie resonances. We showed that using a nanopatch design on top of the cavity design can lead to a polarization-insensitive absorption above 0.8 up to $\lambda=$ $565 \mathrm{~nm}$. Moreover, our calculations estimated a watersplitting photocurrent of $10.6 \mathrm{~mA} \mathrm{~cm}{ }^{-2}$ that is quite close to the maximum achievable theoretical value of a hematite-based photoanode. The proposed design strategy is not only applicable for water-splitting application but also provides an elegant design route for the design of other highly efficient ultrathin optoelectronic devices.

Funding Information This work is supported by the Scientific and Technological Research Council of Turkey (TUBITAK), grant number 215Z249. This work is supported by the projects DPT-HAMIT and TUBITAK under Project Nos. 113E331, 114E374, and 115F560. One of the authors (E. O.) also acknowledges partial support from the Turkish Academy of Sciences.

\section{References}

1. Soukoulis CM, Wegener M (2011) Past achievements and future challenges in the development of three-dimensional photonic metamaterials. Nat Photonics 523

2. Ozbay E (2006) Plasmonics : Merging Photonics and Electronics at Nanoscale Dimensions. Science (80- ) 311:189

3. Yu N, Capasso F (2014) Flat optics with designer metasurfaces. Nat Mater 13:139

4. Ghobadi A, Hajian H, Butun B, Ozbay E (2018) Strong LightMatter Interaction in Lithography-Free Planar Metamaterial Perfect Absorbers. ACS Photonics 5:4203

5. Hajian H, Ghobadi A, Butun B, Ozbay E (2019) Active metamaterial nearly perfect light absorbers: a review [Invited]. J Opt Soc Am B 36:F131

6. Ghobadi A, Hajian H, Butun B, Ozbay E (2019) Strong Interference in Planar Multilayer Perfect Absorbers. IEEE Nanotechnol Mag 13:34

7. Ghobadi A, Ulusoy Ghobadi TG, Karadas F, Ozbay E (2019) Semiconductor Thin Film Based Metasurfaces and Metamaterials for Photovoltaic and Photoelectrochemical Water Splitting Applications. Adv Opt Mater 7:1900028

8. Ji C, Lee K, Xu T, Zhou J, Park HJ, Guo LJ (2017) Engineering Light at the Nanoscale : Structural Color Filters and Broadband Perfect Absorbers. Adv Opt Mater 5:1700368

9. Cui Y, Fung KH, Xu J, Ma H, Jin Y, He S, Fang NX (2012) Ultrabroadband light absorption by a sawtooth anisotropic metamaterial slab. Nano Lett 12:1443

10. Ghobadi A, Hajian H, Gokbayrak M, Butun B, Ozbay E (2019) Broadband polarization-independent resonant light absorption using ultrathin plasmonic super absorbers. Nanophotonics 8:823

11. Ghobadi A, Hajian H, Soydan MC, Butun B, Ozbay E (2019) Omnidirectional, broadband light absorption using large-area, ultrathin lossy metallic film coatings. Sci Rep 9:1

12. Yildirim DU, Ghobadi A, Soydan MC, Atesal O, Toprak A, Caliskan MD, Ozbay E (2019) Ultra-broadband, wide angle absorber utilizing metal insulator multilayers stack with a multi- thickness metal surface texture. ACS Photonics 6:1812 
13. Hajian H, Ghobadi A, Butun B, Ozbay E (2018) Visible light nearly perfect absorber: an optimum unit cell arrangement for near absolute polarization insensitivity. Opt Express 26:16940

14. Yildirim DU, Ghobadi A, Ozbay E (2018) Disordered Nanohole Patterns in Metal-Insulator Multilayer for Ultra-broadband Light Absorption: Atomic Layer Deposition for Lithography Free Highly repeatable Large Scale Multilayer Growth. Sci Rep 8:1

15. Soydan MC, Ghobadi A, Yildirim DU, Behcet V, Ekmel E (2019) Large-area, Lithography-free super absorbers and color filters at visible frequencies using ultrathin metallic films. Plasmonics 1:1

16. Aydin K, Ferry VE, Briggs RM, Atwater HA (2011) Structural Coloring of Glass Using Dewetted Nanoparticles and Ultrathin Films of Metals. Nat Commun 2:1

17. Li Z, Palacios E, Butun S, Kocer H, Aydin K (2015) Broadband light absorption enhancement in thin-film silicon solar cells. Sci Rep 5:1

18. Ghobadi A, Dereshgi SA, Hajian H, Bozok B, Butun B (2017) Bismuth-based metamaterials: From narrowband reflective color filter to extremely broadband near perfect absorber. Sci Rep 7:4755

19. Ghobadi A, Hajian H, Gokbayrak M, Dereshgi SA, Toprak A, Butun B, Ozbay E (2017) Lithography-Free Planar Band-Pass Reflective Color Filter Using Series Connection of Cavities. Opt Express 25:27624

20. Ghobadi A, Hajian H, Dereshgi SA, Bozok B, Butun B, Ozbay E (2017) Disordered and Densely Packed ITO Nanorods as an Excellent Lithography-Free Optical Solar Reflector Metasurface. Sci Rep 7:1

21. Li Z, Butun S, Aydin K (2015) Tunable, omnidirectional , and nearly perfect resonant absorptions by a graphene-hBN- based hole array metamaterial. ACS Photonics 2:183

22. Yu R, Mazumder P, Borrelli NF, Carrilero A, Ghosh DS, Maniyara RA, Baker D, García De Abajo FJ, Pruneri V (2016) Near-absolute polarization insensitivity in graphene based ultra-narrowband perfect visible light absorber. ACS Photonics 3:1194

23. Wang W, Wu S, Reinhardt K, Lu Y, Chen S (2010) All CeramicBased Metal-Free Ultra-broadband Perfect Absorber. Nano Lett 10: 2012

24. Liu N, Mesch M, Weiss T, Hentschel M, Giessen H, Institut $P$ (2010) Infrared Perfect Absorber and Its Application As Plasmonic Sensor. Nano Lett 10:2342

25. Tittl A, Mai P, Taubert R, Dregely D, Liu N, Giessen H (2011) Palladium-based plasmonic perfect absorber in the visible wavelength range and its application to hydrogen sensing. Nano Lett 11:4366

26. Yildirim DU, Ghobadi A, Soydan MC, Gokbayrak M, Toprak A, Butun B, Ozbay E (2019) Colorimetric and Near-Absolute Polarization-Insensitive Refractive-Index Sensing in All-Dielectric Guided-Mode Resonance Based Metasurface. J Phys Chem C 123: 19125

27. Lee T, Jang J, Jeong H, Rho J (2018) Plasmonic- and dielectricbased structural coloring: from fundamentals to practical applications. Nano Converg 5:1

28. Pu M, Ma X, Li X, Guo Y, Luo X (2017) Merging plasmonics and metamaterials by two-dimensional subwavelength structures. J Mater Chem C 5:4361

29. Hajian H, Ghobadi A, Butun B, Ozbay E (2017) Nearly perfect resonant absorption and coherent thermal emission by hBN-based photonic crystals. Opt Express 25

30. Lee BJ, Fu CJ, Zhang ZM (2005) Coherent thermal emission from one-dimensional photonic crystals. Appl Phys Lett 87:071904

31. Zheludev NI (2010) The road ahead for metamaterials. Science (80.):328:582

32. Enoch S, Tayeb G, Sabouroux P, Guérin N, Vincent P (2002) A Metamaterial for Directive Emission. Phys Rev Lett 89:1

33. Vora A, Gwamuri J, Pala N, Kulkarni A, Pearce JM, Güney DO (2014) Exchanging ohmic losses in metamaterial absorbers with useful optical absorption for photovoltaics. Sci Rep 4:1
34. Wu C, Neuner B, John J, Milder A, Zollars B, Savoy S, Shvets G (2012) Metamaterial-based integrated plasmonic absorber/emitter for solar thermo-photovoltaic systems. J Opt 14:1

35. Molesky S, Dewalt CJ, Jacob Z (2013) High temperature epsilonnear-zero and epsilon-near-pole metamaterial emitters for thermophotovoltaics. Opt Express 21:A96

36. Guo CF, Sun T, Cao F, Liu Q, Ren Z (2014) Metallic nanostructures for light trapping in energy-harvesting devices. Light Sci Appl 42:1

37. Chen X, Jia B, Zhang Y, Gu M (2013) Exceeding the limit of plasmonic light trapping in textured screen-printed solar cells using $\mathrm{Al}$ nanoparticles and wrinkle-like graphene sheets. Light Sci. Appl. 2:1

38. Su YH, Ke YF, Cai SL, Yao QY (2012) Surface plasmon resonance of layer-by-layer gold nanoparticles induced photoelectric current in environmentally-friendly plasmon-sensitized solar cell. Light Sci. Appl. 1:2

39. Li W, Valentine J (2014) Metamaterial perfect absorber based hot electron photodetection. Nano Lett 14:3510

40. Ghobadi A, Demirag Y, Hajian H, Toprak A, Butun B, Ozbay E (2019) Spectrally Selective Ultrathin Photodetectors Using Strong Interference in Nanocavity Design. IEEE Electron Device Lett 40:1

41. Li W, Coppens ZJ, Vázquez L, Wang W, Govorov AO, Valentine J (2015) Circularly polarized light detection with hot electrons in chiral plasmonic metamaterials. Nat Commun 6:1

42. Zhao F, Zhou X, Shi Y, Qian X, Alexander M, Zhao X, Mendez S, Yang R, Qu L, Yu G (2018) Highly efficient solar vapour generation via hierarchically nanostructured gels. Nat Nanotechnol 13:489

43. Lee J, Mubeen S, Ji X, Stucky GD, Moskovits M (2012) Plasmonic photoanodes for solar water splitting with visible light. Nano Lett 12:5014

44. Ghobadi TGU, Ghobadi A, Ozbay E, Karadas F (2018) Strategies for Plasmonic Hot-Electron-Driven Photoelectrochemical Water Splitting. ChemPhotoChem 2:161

45. Ghobadi A, Ghobadi TGU, Karadas F, Ozbay E (2018) Angstrom Thick ZnO Passivation Layer to Improve the Photoelectrochemical Water Splitting Performance of a TiO2 Nanowire Photoanode: The Role of Deposition Temperature. Sci Rep 8:16322

46. Ni M, Leung MKH, Leung DYC, Sumathy K (2007) A review and recent developments in photocatalytic water-splitting using $\mathrm{TiO} 2$ for hydrogen production. Renew Sust Energ Rev 11:401

47. Moniz SJA, Shevlin SA, Martin DJ, Guo ZX, Tang J (2015) Visible-light driven heterojunction photocatalysts for water splitting-a critical review. Energy Environ Sci 8:731

48. Ahmad H, Kamarudin SK, Minggu LJ, Kassim M (2015) Hydrogen from photo-catalytic water splitting process: A review. Renew Sust Energ Rev 43:599

49. Kudo A, Miseki Y (2009) Heterogeneous photocatalyst materials for water splitting. Chem Soc Rev 38:253

50. Shen S, Lindley SA, Chen X, Zhang JZ (2016) Hematite heterostructures for photoelectrochemical water splitting: Rational materials design and charge carrier dynamics. Energy Environ Sci 9:2744

51. Sivula K, Le Formal F, Grätzel M (2011) Solar water splitting: Progress using hematite $(\alpha-\mathrm{Fe} 2 \mathrm{O} 3)$ photoelectrodes. ChemSusChem 4:432

52. Tamirat AG, Rick J, Dubale AA, Su WN, Hwang BJ (2016) Using hematite for photoelectrochemical water splitting: A review of current progress and challenges. Nanoscale Horiz 1:243

53. Mao A, Shin K, Kim JK, Wang DH, Han GY, Park JH (2011) Controlled synthesis of vertically aligned hematite on conducting substrate for photoelectrochemical cells: Nanorods versus nanotubes. ACS Appl Mater Interfaces 3:1852

54. Lin Y, Zhou S, Sheehan SW, Wang D (2011) Nanonet-Based hematite heteronanostructures for efficient solar water splitting. J Am Chem Soc 133:2398

55. Goncalves RH, Lima BHR, Leite ER (2011) Magnetite colloidal nanocrystals: A facile pathway to prepare mesoporous hematite thin 
films for photoelectrochemical water splitting. J Am Chem Soc 133:6012

56. Wang G, Ling Y, Wheeler DA, George KEN, Horsley K, Heske C, Zhang JZ, Li Y (2011) Back electron-hole recombination in hematite photoanodes for water splitting. Nano Lett 11:3503

57. Li L, Yu Y, Meng F, Tan Y, Hamers RJ, Jin S (2012) Review of SnDoped hematite nanostructures for photoelectrochemical water splitting. Nano Lett 12:724

58. Mohapatra SK, John SE, Banerjee S, Misra M (2009) Nanostructured hematite thin films produced by spin-coating deposition solution: Application in water splitting. Chem Mater 21:3048

59. Le Formal F, Pendlebury SR, Cornuz M, Tilley SD, Grätzel M, Durrant JR (2014) Activation of hematite nanorod arrays for photoelectrochemical water splitting. J Am Chem Soc 136:2564

60. Ling Y, Li Y (2014) Light-induced water splitting with hematite: Improved nanostructure and iridium oxide catalysis. Part Part Syst Charact 31:1113

61. Souza FL, Lopes KP, Nascente PAP, Leite ER (2009) Formation of iron oxide nanoparticles for the photooxidation of water: Alteration of finite size effects from ferrihydrite to hematite. Sol Energy Mater Sol Cells 93:362

62. Morrish R, Rahman M, MacElroy JMD, Wolden CA (2011) Translucent thin film Fe2O3 photoanodes for efficient water splitting by sunlight: Nanostructure-directing effect of Si-doping. ChemSusChem 4:474

63. Tilley SD, Cornuz M, Sivula K, Grtzel M (2010) Passivating surface states on water splitting hematite photoanodes with alumina overlayers. Angew Chem Int Ed 49:6405

64. Schwaminger SP, Surya R, Filser S, Wimmer A, Weigl F, FragaGarcía P, Berensmeier S (2017) Facile synthesis of highly photoactive $\alpha$-Fe2O3-based films for water oxidation. Sci Rep 7:1

65. Cesar I, Kay A, Martinez JAG, Grätzel M (2006) Facile solution synthesis of $\alpha-\mathrm{FeF} 3 \cdot 3 \mathrm{H} 2 \mathrm{O}$ nanowires and their conversion to $\alpha$ $\mathrm{Fe} 2 \mathrm{O} 3$ nanowires for photoelectrochemical application. J Am Chem Soc 128:4582

66. Le Formal F, Tétreault N, Cornuz M, Moehl T, Grätzel M, Sivula K (2011) Water photooxidation by smooth and ultrathin R-Fe2O3 nanotube arrays. Chem Sci 2:737

67. Liu J, Cai YY, Tian ZF, Ruan GS, Ye YX, Liang CH, Shao GS (2014) Highly oriented Ge-doped hematite nanosheet arrays for photoelectrochemical water oxidation. Nano Energy 9:282

68. Peerakiatkhajohn P, Yun JH, Chen H, Lyu M, Butburee T, Wang L (2016) Stable Hematite Nanosheet Photoanodes for Enhanced Photoelectrochemical Water Splitting. Adv Mater 28:6405

69. Bu X, Wang G, Tian Y (2017) Foreign In3+ treatment improving the photoelectrochemical performance of a hematite nanosheet array for water splitting. Nanoscale 9:17513

70. Liu J, Liang C, Zhang H, Tian Z, Zhang S (2012) General strategy for doping impurities (Ge, Si, Mn, Sn, Ti) in hematite nanocrystals. J Phys Chem C 116:4986

71. Ji M, Cai J, Ma Y, Qi L (2016) Controlled Growth of Ferrihydrite Branched Nanosheet Arrays and Their Transformation to Hematite Nanosheet Arrays for Photoelectrochemical Water Splitting. ACS Appl Mater Interfaces 8:3651

72. Wang KX, Yu Z, Liu V, Brongersma ML, Jaramillo TF, Fan S (2014) Nearly Total Solar Absorption in Ultrathin Nanostructured Iron Oxide for Efficient Photoelectrochemical Water Splitting. ACS Photonics 1

73. Qiu Y, Leung SF, Zhang Q, Hua B, Lin Q, Wei Z, Tsui KH, Zhang Y, Yang S, Fan Z (2014) Efficient photoelectrochemical water splitting with ultrathin films of hematite on three-dimensional nanophotonic structures. Nano Lett 14:2123

74. Li J, Qiu Y, Wei Z, Lin Q, Zhang Q, Yan K, Chen H, Xiao S, Fan Z, Yang S (2014) A three-dimensional hexagonal fluorine-doped tin oxide nanocone array: A superior light harvesting electrode for high performance photoelectrochemical water splitting. Energy Environ Sci 7:3651
75. Li J, Cushing SK, Zheng P, Meng F, Chu D, Wu N (2013) Plasmoninduced photonic and energy-transfer enhancement of solar water splitting by a hematite nanorod array. Nat Commun 4(2651):1

76. Pincella F, Isozaki K, Miki K (2014) A visible light-driven plasmonic photocatalyst. Light Sci Appl 3:1

77. Gao H, Liu C, Jeong HE, Yang P (2012) Plasmon-enhanced photocatalytic activity of iron oxide on gold nanopillars. ACS Nano 6:234

78. Wang L, Wang L, Hu H, Truong N, Zhang Y, Schmuki P, Bi Y (2017) Plasmon-Induced Hole-Depletion Layer on Hematite Nanoflake Photoanodes for Highly Efficient Solar Water Splitting. Nano Energy 35:171

79. Liu D, Bierman DM, Lenert A, Yu H-T, Yang Z, Wang EN, Duan Y-Y (2015) Ultrathin planar hematite film for solar photoelectrochemical water splitting. Opt Express 23:A1491

80. Dotan H, Kfir O, Sharlin E, Blank O, Gross M, Dumchin I, Ankonina G, Rothschild A (2012) Resonant light trapping in ultrathin films for water splitting. Nat Mater 12:158

81. Kim SJ, Thomann I, Park J, Kang JH, Vasudev AP, Brongersma ML (2014) Light trapping for solar fuel generation with Mie resonances. Nano Lett 14:1446

82. Liu D, Yu H, Duan Y, Li Q, Xuan Y (2016) New Insight into the Angle Insensitivity of Ultrathin Planar Optical Absorbers for Broadband Solar Energy Harvesting. Sci Rep 6:32515

83. Liu D, Yu H, Yang Z, Duan Y (2016) Ultrathin planar broadband absorber through effective medium design. Nano Res 9:2354

84. Mirshafieyan SS, Guo J (2014) Silicon colors: spectral selective perfect light absorption in single layer silicon films on aluminum surface and its thermal tunability. Opt Express 22:31545

85. Park J, Kang J, Vasudev AP, Schoen DT, Kim H, Hasman E, Brongersma ML (2014) Omnidirectional Near-Unity Absorption in an Ultrathin Planar Semiconductor Layer on a Metal Substrate. ACS Photon 1:812

86. Yoo YJ, Lim JH, Lee GJ, Jang KI, Song YM (2017) Ultra-thin films with highly absorbent porous media fine-tunable for coloration and enhanced color purity. Nanoscale 9:2986

87. Kats MA, Blanchard R, Genevet P, Capasso F (2012) Nanometre optical coatings based on strong interference effects in highly absorbing media. Nat Mater 12:20

88. Palik ED (1998) Handbook of optical constants of solids. Vol. 3 (Academic press

89. Ghobadi A, Hajian H, Rashed AR, Butun B, Ozbay E (2018) Tuning the metal filling fraction in metal-insulator-metal ultrabroadband perfect absorbers to maximize the absorption bandwidth. Photon Res 6:168

90. In Lumerical Solut. Inc. (n.d.) http://Www.Lumerical.Com/TcadProducts/Fdtd/

91. Xie X, Li K, De Zhang W (2016) Photoelectrochemical properties of Ti-doped hematite nanosheet arrays decorated with CdS nanoparticles. RSC Adv 6:74234

92. Lei R, Ni H, Chen R, Gu H, Zhang B, Zhan W (2018) Hydrothermal synthesis of CdS nanorods anchored on $\alpha$ $\mathrm{Fe} 2 \mathrm{O} 3$ nanotube arrays with enhanced visible-light-driven photocatalytic properties. J Colloid Interface Sci 514:496

93. Ghobadi A, Ulusoy TG, Garifullin R, Guler MO, Okyay AK (2016) A Heterojunction Design of Single Layer Hole Tunneling ZnO Passivation Wrapping around $\mathrm{TiO} 2$ Nanowires for Superior Photocatalytic Performance. Sci Rep 6:30587

94. Ghobadi A, Yavuz HI, Ulusoy TG, Icli KC, Ozenbas M, Okyay AK (2015) Enhanced Performance of Nanowire-Based All-TiO2 Solar Cells using Subnanometer-Thick Atomic Layer Deposited ZnO Embedded Layer. Electrochim Acta 157

Publisher's Note Springer Nature remains neutral with regard to jurisdictional claims in published maps and institutional affiliations. 